\title{
eJRIEPS
}

Ejournal de la recherche sur l'intervention en éducation physique et sport

$19 \mid 2010$

Varia

\section{Analyse de l'accompagnement du stage en responsabilité au Québec et en France : un regard sur les entretiens post-leçons à l'aide de l'écologie de la classe}

Jérôme Leriche, Jean-François Desbiens, Jean-Paul Dugal et Chantal Amade-Escot

\section{(2) OpenEdition}

Journals

Édition électronique

URL : http://journals.openedition.org/ejrieps/5443

DOI : 10.4000/ejrieps.5443

ISSN : 2105-0821

Éditeur

ELLIADD

Référence électronique

Jérôme Leriche, Jean-François Desbiens, Jean-Paul Dugal et Chantal Amade-Escot, « Analyse de l'accompagnement du stage en responsabilité au Québec et en France : un regard sur les entretiens post-leçons à l'aide de l'écologie de la classe », eJRIEPS [En ligne], 19 | 2010, mis en ligne le 01 janvier 2010, consulté le 08 janvier 2021. URL : http://journals.openedition.org/ejrieps/5443 ; DOI : https:// doi.org/10.4000/ejrieps.5443

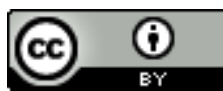

La revue eJRIEPS est mise à disposition selon les termes de la Creative Commons Attribution 4.0 International License. 
Analyse de l'accompagnement du stage en responsabilité au Québec et en

France : un regard sur les entretiens post-leçons à l'aide de l'écologie de la classe

Jérôme Leriche*, Jean-François Desbiens*, Jean-Paul Dugal ${ }^{* *}$, Chantal Amade-Escot** GRIEFPAP / DiDiST-CRÉFI-T, Cotutelle Québec-France, *Université de Sherbrooke (Québec) / **Université Paul-Sabatier Toulouse (France)

\section{Résumé}

La formation des enseignants d'éducation physique au Québec comme en France vise l'acquisition de compétences professionnelles. Les maîtres de stage qui accompagnent les stagiaires durant les stages en responsabilité ont un rôle déterminant. Dans cette recherche, nous nous intéressons, dans une perspective d'éducation comparée, aux pratiques d'accompagnement mises en place par un maître de stage au Québec et un autre en France lors du stage obligatoire d'une durée de 7 semaines au Québec et de 7 mois en France. Nous analysons à l'aide du cadre théorique de l'écologie de la classe les entretiens post-leçons qui sont au cœur des pratiques d'accompagnement afin de quantifier et de qualifier le contenu des propos échangés entre le maître de stage et son stagiaire dans les deux pays. Pour saisir la singularité de ces deux contextes, nous complétons ces données en caractérisant la fréquence et le contenu des pratiques d'accompagnement mises en place à partir d'entretiens téléphoniques avec les deux stagiaires. Nos résultats montrent que malgré des contextes différents, certaines modalités d'accompagnement sont fort semblables entre le Québec et la France, bien que des spécificités marquées par les systèmes de formation nationaux soient observées.

Le système de recrutement et de formation des enseignants va vivre en France une réforme majeure en 2010, ce qui devrait modifier le rôle et la forme des stages en responsabilité effectués par les étudiants. Orientés vers une professionnalisation du métier d'enseignant, ces stages ont actuellement une durée de 7 semaines au Québec et de 7 mois en France et sont encadrés par des maîtres de stage. Dans cet article nous nous intéressons aux pratiques d'accompagnement mises en œuvre par ces derniers dans les deux institutions afin d'apporter une contribution à la réflexion pour les réformes de demain en matière de formation à l'enseignement.

Jusqu'à présent, de nombreux gouvernements, dont celui du Québec et de la France, ont adopté une formation des enseignants d'éducation physique (EP) basée sur l'acquisition 
de compétences professionnelles dûment répertoriées. II est également supposé que l'alternance de périodes de formation « théoriques » et de stages dans les établissements scolaires permettrait de mieux répondre aux exigences de la profession enseignante (MEN, 2002 ; MELS, 2001). La majorité des chercheurs s'intéressant à la formation des enseignants soutiennent que les compétences professionnelles se développent en situation de pratique (Ria, Sève, Durand et Bertone, 2004; Roux-Pérez, 2006; Serres, Ria, Adé et Sève, 2006; Wittorski, 2004). Ce point de vue est relayé dans les textes ministériels encadrant les parcours de formation des enseignants (BOEN $n^{\circ} 1$ du 04/01/07; MELS, 2001 ). Plusieurs auteurs s'accordent pour dire que le montage et le rodage progressifs de compétences professionnelles sont justement facilités par un aller-retour entre les savoirs théoriques et pratiques en relation avec l'expérience (Brien, 1994; Serres et al., 2006). Les stages en formation sont précisément les périodes où l'étudiant se retrouve au cœur des tensions entre les valeurs et les savoirs véhiculés par l'institution de formation et ceux du milieu de pratique. Cependant, les stages ne suffisent pas à eux seuls à favoriser le développement de compétences professionnelles en enseignement. Pour qu'un stagiaire retire les bénéfices de son expérience, il faut qu'il soit capable de l'interpréter, de prendre un certain recul et de faire des liens entre les différents savoirs et habiletés qu'il a mobilisés dans les situations vécues. Le succès de cette entreprise semble fortement associé aux pratiques d'accompagnement mises en place par le maître de stage (Banville, 2006).

\section{La formation de terrain : une alternance interrogée}

Le choix d'une formation des enseignants d'EP professionnalisante au Québec et en France a eu pour effet de modifier en profondeur les cursus de formations. Au Québec, cette orientation s'est concrétisée par la mise en place d'un programme de formation des maîtres organisé autour de 12 compétences professionnelles à développer en formation (MELS, 2001). En France, un nouveau programme structuré autour de 10 compétences professionnelles a été mis en place dans les IUFM à la rentrée de septembre 2007 (BOEN no 1 du 04/01/07).

1. 1. Les nouveaux enjeux de la formation de terrain : le point de vue institutionnel Dans le but de développer des compétences professionnelles, les systèmes québécois et français se basent sur un processus de formation par l'alternance. Ainsi, les stages prennent de plus en plus de place au fur et à mesure que les étudiants d'EP s'approchent de la fin de leur formation. Ces périodes de stage représentent 700 heures sur quatre ans au Québec (MELS, 2001), dont une grande partie est concentrée sur les deux dernières 
années ( $3^{e}$ et $4^{\mathrm{e}}$ année universitaire). En France, les étudiants réalisent 360 heures de stage durant l'année de professionnalisation qui correspond à la dernière année de formation en IUFM (5 $5^{\mathrm{e}}$ année universitaire) (BOEN no 9 du 01/03/2007).

Au Québec, le rapport d'évaluation des programmes de 2002 souligne que, dans un contexte de formation professionnelle, le rôle du maître de stage est fondamental et lourd de responsabilités, car il doit accueillir, conseiller, soutenir et évaluer le stagiaire tout en l'inscrivant dans une démarche réflexive (MELS, 2002, p. 51). En France, le maître de stage doit aider le stagiaire à adapter ses pratiques à la réalité du terrain et à les mobiliser dans la perspective du développement des 10 compétences professionnelles. Pour aider les maîtres de stage à faire face à ces nouveaux enjeux, des périodes de formation sont mises en place dans chacune des institutions. Cependant, malgré une nette amélioration au cours des dernières années, ces formations semblent être problématiques autant au Québec qu'en France. Dans le premier cas, 58,6 \% des maîtres de stage ont reçu une formation en 2002 et seulement 17,8 \% les 30 heures de formation minimale prévues (MELS, 2002, p. 29-30). En France, les maîtres de stage sont essentiellement soutenus par des textes réglementaires ; ils reçoivent peu de formation et le cahier des charges est laissé à la discrétion des instituts de formation des maîtres (Lacoste, Loarer et Monnanteuil, 2007). Dans les institutions québécoises et françaises, les problèmes en lien avec l'accompagnement des stagiaires en fin de formation sont également remis en question par des rapports gouvernementaux (COFPE, 2005, 2006; MEN, 2008). Les interrogations et constats que suscitent ces rapports mettent en évidence la nécessité d'une réflexion sur l'accompagnement au cours de la formation en stage des enseignants d'EP tant au Québec qu'en France.

1. 2. Des questionnements relayés par les chercheurs

Plusieurs recherches en éducation (Chaliès et Durand, 2000; Leclerc, 2004) et en EP (Banville, 2006; Ria et al., 2004; Roux-Perez, 2006) indiquent que les stagiaires ont de la difficulté à analyser de manière dialectique les connaissances issues de la formation théorique avec les expériences vécues en stage. Ce processus nécessite un accompagnement pour amener le stagiaire à développer les compétences professionnelles attendues (Barbier, 2006). L'accent mis sur les stages en formation initiale confère donc aux enseignants chargés de l'accompagnement des stagiaires des missions importantes. À ce sujet, Banville (2006) qualifie les maîtres de stage de joueurs clefs («key players ») dans le bon déroulement de l'expérience de terrain des stagiaires. Le rôle majeur des maîtres de stage est également souligné par 164 stagiaires français en EP qui, à hauteur de $49 \%$, les identifient comme le premier facteur responsable de leur 
amélioration pédagogique (Roux-Pérez, 2006). Cependant, le changement de posture que suppose le passage du rôle d'enseignant à celui de maître de stage semble être délicat. Cela implique l'acquisition d'une certaine culture de la formation des enseignants, mais également de passer d'une pratique d'enseignant à une pratique d'accompagnateur. Ainsi, dans leur revue de la documentation internationale basée sur plus de 270 publications scientifiques traitant du tutorat en formation initiale des enseignants, Chaliès et Durand qualifient les pratiques d'accompagnement d'énigmatiques (2000, p. 163) et soulignent que ces dernières appellent un effort de compréhension. Les maîtres de stage jouent un rôle important, notamment lors des stages en responsabilité qui précèdent la titularisation des jeunes enseignants. À ce sujet, Lacoste et al. (2007) déclarent que le stage en responsabilité (le dernier stage de la formation) constitue l'élément structurant dans le processus en alternance et un élément central de la formation des enseignants.

Les questionnements soulevés par les ministères québécois et français chargés de la formation des enseignants d'EP et les résultats de la recherche au sujet de la formation professionnelle nous invitent à étudier les formes d'accompagnement des stagiaires en fin de formation de ces deux institutions: quelles sont les modalités et les contenus des pratiques d'accompagnement mises en place par des maîtres de stage au Québec et en France ? Sont-elles liées aux contextes particuliers de ces deux systèmes de formation ? Plus précisément, dans une perspective d'éducation comparée entre le Québec et la France, existe-t-il des pratiques d'accompagnement originales permettant d'enrichir la réflexion sur la formation des enseignants dans ces deux contextes ? Mais avant, que disent les recherches portant sur les pratiques d'accompagnement mises en place par les maîtres de stage dans les stages en responsabilité ?

\section{L'accompagnement des stagiaires : état de la question}

2. 1. Fréquence et intensité des pratiques d'accompagnement en stage

À la fin des années 1980, Tannehill et Zakrajsek se sont intéressés aux pratiques d'accompagnement de stagiaires en enseignement de l'EP et ils ont montré que la fréquence et la durée de l'accompagnement des stagiaires par les maîtres de stage diminuaient au fur et à mesure de l'avancée du stage jusqu'à une autonomie complète du stagiaire dans les dernières semaines (Tannehill et Zakrajsek, 1988). Cette tendance est également observée par Banville (2006) qui a montré que le nombre de commentaires des maîtres de stage baissait de $45 \%$ entre un entretien dans les premières semaines du stage et celui dans les dernières. Les résultats de ces recherches soulignent également la grande diversité des modalités des pratiques d'accompagnement des stagiaires, même si 
elles observent que la plupart d'entre elles se structurent autour des entretiens postleçons.

2. 2. L'entretien post-leçon : la pierre angulaire des pratiques d'accompagnement Une synthèse des recherches sur les pratiques d'accompagnement en EP réalisées par Coleman et Mitchel (2000) montre que l'observation de la leçon par les maîtres de stage suivie d'un entretien post-leçon (aussi appelé entretien-conseil) avec le stagiaire sont deux piliers de l'accompagnement que l'on retrouve dans les six études qu'ils ont analysées. À ce sujet, une étude portant sur 12 entretiens post-leçons entre six maîtres de stage et leurs stagiaires (deux chacun) pendant un stage en responsabilité de sept semaines aux États-Unis montre que les thématiques traitées le plus souvent sont la gestion de classe (35,5 \% du temps) et la planification de la séance (32,6\% du temps) (Banville, 2006). Le cadre utilisé pour l'analyse de ces entretiens post-leçons était les neuf compétences professionnelles du référentiel étasunien (Banville, 2006). D’autre part, Dugal et AmadeEscot (2004) font ressortir que dans les entretiens post-leçons analysés chez trois dyades françaises (maîtres de stage - stagiaire), les aspects reliés à l'organisation et la gestion de la classe sont majoritaires. En se basant sur un cadre théorique et conceptuel inspiré de Bru (1993) et Altet (1994), ces chercheurs constatent que plus de la moitié des thèmes de discussion entre les maîtres de stage et leurs stagiaires sont associés à la catégorie « organisation » alors que les catégories « intervention auprès des élèves » et « contenus enseignés » se partagent le temps restant des entretiens post-leçons.

2. 3. Le sens donné par les maîtres de stage à leurs pratiques d'accompagnement Clarke (2006) a suivi et analysé les entretiens pré et post-leçons de cinq dyades canadiennes pendant 13 semaines de stage à partir de la technique du rappel stimulé avec le maître de stage. Cette étude cherchait davantage à comprendre le sens que les maîtres de stage donnaient à leurs pratiques d'accompagnement qu'à analyser en profondeur les échanges avec le stagiaire. II ressort de cette étude que le fait d'analyser la pratique d'enseignement d'un stagiaire amène les maîtres de stage à réfléchir sur leurs propres pratiques en tant qu'enseignant d'EP. Les échanges lors de ces entretiens prennent certes naissance à partir d'incidents relevés dans la séance du stagiaire, mais qu'ils restent d'abord et avant tout liés aux thématiques chères au maître de stage. Le suivi d'un stagiaire et de son maître de stage pendant le stage en responsabilité d'un an qui clôture la formation des enseignants d'EP en France a permis de rendre compte du sens donné par le stagiaire et son maître de stage aux expériences vécues en classe et au regard porté sur les évènements survenus au cours de l'entretien post-leçon. II ressort de cette étude que le maître de stage a tendance à adopter une posture plutôt directive 
dans ses recommandations et que le stagiaire est amené à justifier constamment ses pratiques (Bertone, Chaliès, Clarke et Méard, 2006). Cette recherche fait aussi apparaître les difficultés de communication qui peuvent exister entre le maître de stage et son stagiaire au cours des entretiens post-leçons.

Ce bref état de la question montre que les recherches sur les pratiques d'accompagnement mises en place par les maîtres de stage ont été réalisées selon trois orientations. La première cherchait à identifier et analyser la fréquence des pratiques d'accompagnement au cours des stages. La deuxième consistait à analyser le contenu des entretiens post-leçons à partir de catégories prédéterminées. La troisième consistait à identifier le sens que les maîtres de stage et les stagiaires accordent aux pratiques d'accompagnement qui ont lieu au cours de l'entretien post-leçon. D'une manière générale, les études sont menées dans des contextes nationaux, elles portent sur plusieurs dyades. Notre recherche quant à elle s'inscrit dans les deux premières orientations et vise à comparer les pratiques d'accompagnement dans deux contextes différents.

\section{Problématique de l'étude: modalités de l'accompagnement des stagiaires au Québec et en France}

L'originalité de notre étude provient du fait que nous nous proposons d'identifier, de décrire et d'analyser la fréquence et le contenu des pratiques d'accompagnement mises en place par deux maîtres de stage tout au long du stage en responsabilité auprès de leurs stagiaires en EP au Québec et en France. Nous avons choisi de centrer notre recherche sur les entretiens post-leçons tout en prenant en compte la fréquence et la durée des pratiques d'accompagnement en dehors de ces entretiens. Ceci nous permettra d'éclairer, dans une perspective de "formation comparée", les caractéristiques des pratiques d'accompagnement des stagiaires dans deux contextes nationaux de formation des enseignants. Nous n'avons pas jugé pertinent d'utiliser les référentiels de compétences professionnelles pour analyser les entretiens post-leçons parce que d'une part, ils sont différents au Québec et en France et d'autre part, parce que la diversité des compétences attendues dépasse le cadre de l'analyse d'une séance d'EP. En revanche, nous avons considéré que le cadre théorique de l'écologie de la classe (Hastie et Siedentop, 1999, 2006; Siedentop, 2002, ) parce qu'il envisage différents aspects de la pratique enseignante en contexte permet de rendre compte des thématiques traitées dans les entretiens post-leçons (dans les différentes dimensions mises en évidence par la revue de littérature présentée en section 3). Ce choix de cadre théorique nous permet en outre 
d'utiliser une grille d'analyse commune entre le Québec et la France. Étudier les pratiques d'accompagnement des stagiaires à l'aune du cadre théorique de l'écologie de la classe.

Les travaux sur l'écologie de la classe se sont majoritairement développés en Amérique du Nord dans les années 1980. Doyle (1981), qui en est le fondateur s'est appuyé sur les recherches menées par Kounin (1970) pour montrer que l'efficacité des enseignants dépendait de leurs habiletés à contrôler et guider le système complexe de la classe. Selon Doyle (1986), les enseignants doivent adopter des stratégies pour réduire la complexité que représente l'environnement de la classe. Cette manière d'étudier la dynamique de la classe est qualifiée d'écologique, par analogie avec le concept "d'écosystème" utilisé en biologie végétale et animale pour étudier un milieu donné. L'importation de l'approche écologique développée par Doyle au contexte de l'enseignement de l'EP a fait l'objet d'adaptations heuristiques débouchant sur programme de recherche important. On doit à Tousignant et Siedentop (1983) une première formalisation qui, à partir de Doyle (1979, 1981), définit l'enseignement comme une succession de tâches visant l'obtention et le maintien de la coopération des élèves dans l'activité, et ce, dans le temps disponible pour cette activité. De nombreuses recherches, dans la continuité de la thèse de Tousignant (1982), ont permis d'analyser et de comprendre la structure des tâches dans le contexte d'une classe d'EP (Hastie et Siedentop, 1999; Hastie et Siedentop, 2006; Hastie, Sinelnikov, Brock, Sharpe, Eiler et Mowling, 2007; Mc Caughtry, Tischler et Flory, 2008; Siedentop, 2002; Tinning et Siedentop, 1985; Tinning, 2002). Plusieurs concepts ont été développés pour analyser la complexité des interactions en classe. Selon ces auteurs, l'écologie de l'éducation physique s'établit autour de trois systèmes de tâches : le système de tâches d'organisation, le système de tâches d'apprentissage, le système de tâches d'interaction sociale entre les élèves (Tousignant et Siedentop,1983). Le tableau suivant en présente les caractéristiques principales. 
Tableau I. Les systèmes de tâches figurant dans l'écologie de l'EP (inspiré de Siedentop, 1994)

\begin{tabular}{|c|c|c|}
\hline Systèmes & Définition & Exemples \\
\hline $\begin{array}{l}\text { Système de tâches } \\
\text { d'organisation } \\
\text { (STO) }\end{array}$ & $\begin{array}{l}\text {-Relié aux aspects } \\
\text { comportementaux } \\
\text { organisationnels } \\
\text {-Toutes les fonctions qui ne } \\
\text { sont pas reliées à la matière, } \\
\text { mais nécessaires au bon } \\
\text { déroulement de la classe }\end{array}$ & $\begin{array}{l}\text { Lorsqu'un enseignant dit } \\
\text { «Formez quatre équipes pour } \\
\text { jouer au volley-ball en vous } \\
\text { attribuant chacun un numéro de } \\
\text { un à quatre " (Siedentop, 1994, } \\
\text { p. 97) }\end{array}$ \\
\hline $\begin{array}{l}\text { Système de tâches } \\
\text { d'apprentissage } \\
\text { (STA) }\end{array}$ & $\begin{array}{l}\text {-Activité reliée directement à } \\
\text { la matière enseignée en EP } \\
\text {-Les apprentissages que } \\
\text { l'enseignant a l'intention de } \\
\text { faire réaliser aux élèves } \\
\text { pendant leur participation aux } \\
\text { activités proposées }\end{array}$ & $\begin{array}{l}\text { Lorsque l'enseignant dit } \\
\text { “Travaillez deux par deux, à } \\
\text { deux mètres de distance et } \\
\text { conservez le ballon de volley-ball } \\
\text { en jeu en faisant des } \\
\text { manchettes (Siedentop, 1994, } \\
\text { p. 98) }\end{array}$ \\
\hline $\begin{array}{l}\text { Système de tâches } \\
\text { d'interaction } \\
\text { sociale entre les } \\
\text { élèves (STIS) }\end{array}$ & $\begin{array}{l}\text {-Dirigé par les élèves } \\
\text {-Intention des élèves } \\
\text { d'entretenir des relations } \\
\text { sociales avec les autres } \\
\text { participants pendant les } \\
\text { leçons d'EP } \\
\text {-Interfère avec les autres } \\
\text { systèmes de tâches et peut } \\
\text { produire des problèmes pour } \\
\text { l'enseignant }\end{array}$ & $\begin{array}{l}\text { "avoir du plaisir avec un ami } \\
\text { tout en accomplissant de façon } \\
\text { appropriée une tâche } \\
\text { d'apprentissage au volley- } \\
\text { ball (...) se désengager } \\
\text { complètement de la tâche } \\
\text { d'apprentissage pour s'engager } \\
\text { dans des comportements } \\
\text { sociaux dérangeants et } \\
\text { inacceptables (...) " (Siedentop, } \\
\text { 1994, p. 98) }\end{array}$ \\
\hline
\end{tabular}

Dans le cadre de notre étude qui s'attache à analyser le contenu des entretiens postleçons, l'approche conceptuelle proposée par le paradigme de l'écologie de la classe nous semble appropriée, car elle permet de rendre compte de l'ensemble des évènements ayant lieu dans une séance d'EP. Elle permet notamment d'identifier, de décrire et d'analyser les éléments discutés entre le maître de stage et son stagiaire en lien avec la séance d'EP que le maître de stage vient d'observer. Dans le cadre de notre recherche, 
nous nous attacherons à identifier quelle est la part respective des trois systèmes de tâches dans les préoccupations des stagiaires et des maîtres de stage telles qu'elles apparaissent dans leurs interactions au cours des entretiens post-leçons.

\section{Méthodologie}

Cette étude s'inscrit clairement dans une approche qualitative au sens de Van der Maren (1996), car les données qui ont été collectées sont non-métriques et que la démarche est plutôt inductive et le produit de notre recherche se classe dans les théories interprétatives. Néanmoins, dans un but de comparaison nous utiliserons des quasi statistiques (au sens de Maxwell, 2005), c'est-à-dire que nous ferons appel à des composantes quantitatives implicites (par exemple des mesures de rareté ou de fréquence, de faibles ou hautes intensités, etc.). Ce support quantitatif est un moyen pour documenter les pratiques d'accompagnement et doit être perçu comme un ensemble d'indices visant à accroître la validité des analyses en relation avec les caractéristiques des deux contextes étudiés. En effet, nous nous situons dans une recherche de type "étude de cas multiples" (Stake, 2000) selon une approche méthodologique qualifiée d'écologique par Bronfenbrenner $(1980,1981)$ dans le sens où nous avons conçu notre protocole de collecte des données avec comme souci de perturber le moins possible la relation entre les maîtres de stage et leurs stagiaires dans un cas au Québec et dans l'autre en France.

\section{1. Les participants}

La dyade québécoise est composée d'une stagiaire en quatrième année de baccalauréat en enseignement de l'EP effectuant son stage en responsabilité et d'un maître de stage expérimenté. La stagiaire est spécialiste en football et en athlétisme, mais dit avoir des faiblesses dans certains sports collectifs comme le handball. Ses stages précédents se sont bien déroulés et elle n'a pas eu de problème particulier avec ses précédents maîtres de stage. Le maître de stage a précédemment reçu six stagiaires, dont trois en stage en responsabilité au cours des trois dernières années. Ses spécialités sont les sports collectifs (hockey, base-ball et handball). Enfin, il n'a pas reçu de formation spécifique pour devenir maître de stage et nous a dit ne pas avoir reçu d'information pour d'éventuelles formations à ce sujet de la part des institutions qui lui envoyaient les stagiaires.

La dyade française est composée d'un stagiaire en deuxième année d'IUFM et d'un maître de stage expérimenté. Le stagiaire a comme spécialité le football et les sports de raquettes (tennis, badminton) et se sent davantage démuni face à l'enseignement de la gymnastique. Ses stages précédents dans le cadre de l'université et de l'IUFM se sont déroulés sans problème. Son maître de stage a pour sa part déjà accompagné six 
stagiaires. Ses spécialités sont les sports de combat et la gymnastique et il se sent plus faible dans les sports collectifs. II a bénéficié d'une première formation pour assurer la fonction de maître de stage en 2000 et suivi plusieurs stages par la suite (un par année environ).

\section{2. Contextes des stages au Québec et en France}

Notre recherche s'inscrit dans des contextes institutionnels et des traditions de formation différents ayant indéniablement des influences sur les pratiques d'accompagnement mises en place par les maîtres de stage. Le tableau 2 présente de manière globale les caractéristiques des stages et des groupes cibles d'élèves ayant servi à la collecte des données.

Tableau 2 : Récapitulatif des éléments de contexte des études de cas au Québec et en France.

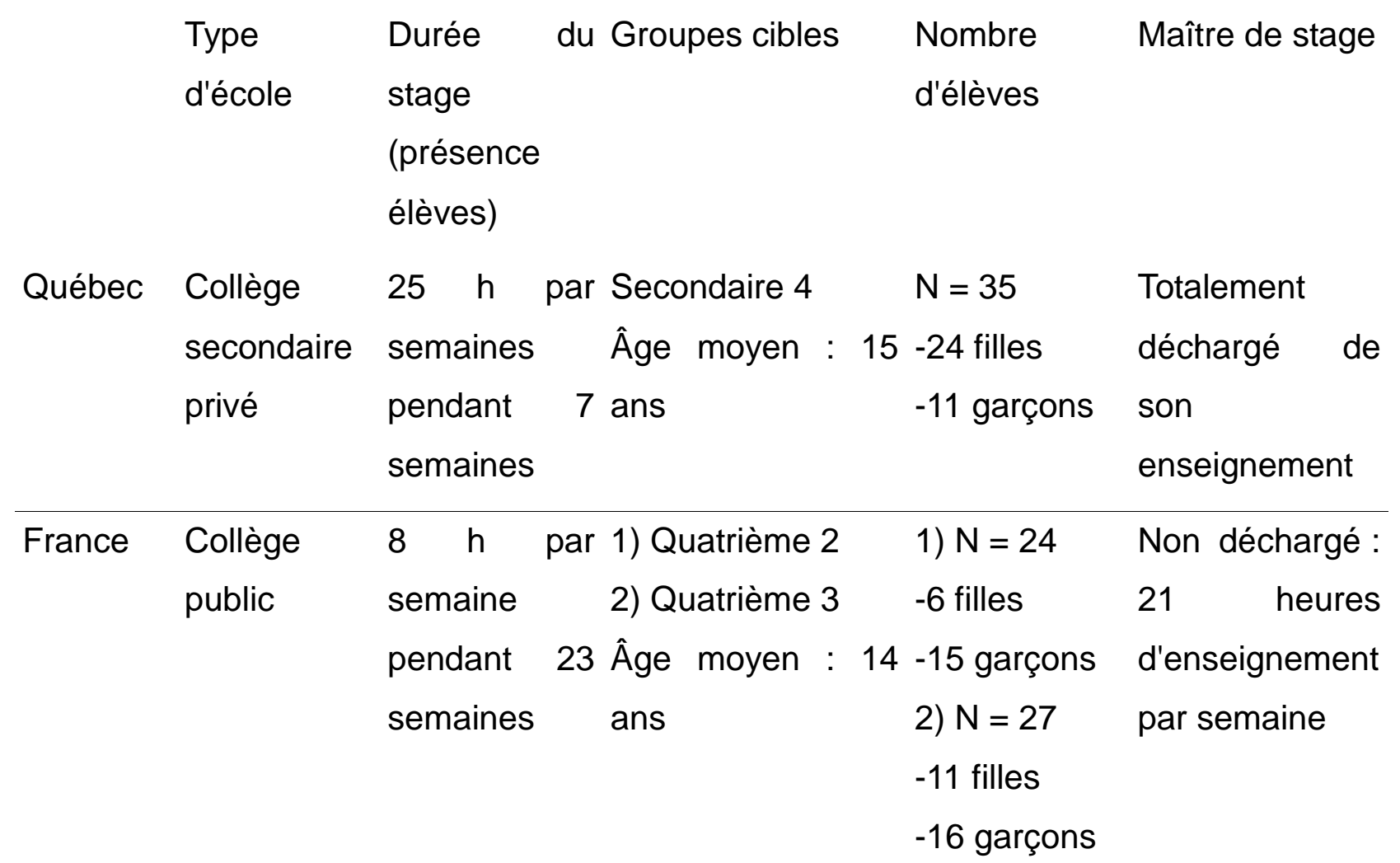

\section{3. Architecture d'ensemble de la collecte des données}

Nous avons suivi deux dyades maîtres de stage - stagiaires pendant toute la durée du stage en responsabilité tel qu'il est programmé dans les contextes de formation respectifs du Québec et de la France. Dans le but de ne pas perturber le déroulement du stage (validité écologique), nous avons collecté des données sur les pratiques d'accompagnement quand elles se présentaient. Notre étude visant prioritairement l'étude des entretiens post-leçons, nous avons collecté les données suivantes: 
- Enregistrements audios des entretiens post-leçons entre les maîtres de stages et les stagiaires suite aux séances enseignées par les stagiaires $(N=3$ au Québec, $N=5$ en France). Ces séances étaient précédemment observées par les maîtres de stage.

- Enregistrement vidéo des séances d'EP précédant les entretiens post-leçons ( $N=3$ au Québec, $N=5$ en France).

Néanmoins, pour situer ces entretiens dans le cadre de l'ensemble des pratiques d'accompagnement en lien avec les contextes de stage québécois et français (voir tableau II), nous avons procédé à un second niveau de collecte des données, ayant un caractère secondaire, mais permettant de prendre en compte des indicateurs plus larges susceptibles de contextualiser les entretiens post-leçons dans la spécificité des systèmes de formation étudiés. Nous avons procédé par entretiens téléphoniques directifs avec les stagiaires ( $N=5$ au Québec, $N=9$ en France) afin d'identifier la fréquence des pratiques d'accompagnement tout au long du stage en dehors des entretiens post-leçons. Les stagiaires devaient répondre à différentes catégories de questions au sujet des rencontres qu'ils avaient pu avoir avec leurs maîtres de stage dans les semaines précédentes (notamment le jour, la date, l'heure la durée et le contenu).

\section{4. Traitement des données}

Le traitement des données s'organise également selon deux niveaux qui correspondent aux méthodes de collecte des données énoncées précédemment. D'une part, les enregistrements des entretiens post-leçons ont été transcrits sous forme de verbatim avant que leur contenu soit analysé à l'aide du logiciel QSR-NVIVO 2. Ce logiciel est conçu pour faciliter le classement et l'analyse des données textuelles à partir de grilles construites par les chercheurs. Les grilles d'analyses utilisées ont été bâties à partir du cadre théorique de l'écologie de la classe et plus particulièrement des trois systèmes de tâches développés par Siedentop (1994). La fidélité des grilles a été évaluée à hauteur de $84.5 \%$ pour la fidélité inter-codeur et à $89.7 \%$ pour la fidélité intra-codeur. De plus, nous avons retenu comme indice macroscopique le temps de parole respectif du maître de stage et du stagiaire lors de chaque entretien, lui-même ventilé selon chacune des catégories de notre grille d'analyse de contenu. Les enregistrements vidéo ont été considérés comme des données «informantes" servant de références pour contextualiser et mieux comprendre les entretiens post-leçons. D'autre part, les données secondaires obtenues à partir des entretiens téléphoniques avec les stagiaires ont été synthétisées à partir des indicateurs de date, durée et lieu des rencontres entre le maître de stage et le stagiaire. 
5. Résultats : éléments contextuels permettant d'éclairer les entretiens postleçons

L'architecture de la collecte des données s'organise en deux niveaux : le premier renvoie aux entretiens post-leçons dont l'étude est au cœur de cette recherche et le second à l'aide des entretiens téléphoniques permet de mieux saisir la spécificité des contextes de stage. Nous avons décidé de présenter dans un premier temps les résultats issus des données secondaires afin de pouvoir porter ensuite un éclairage qui prend en compte les particularités québécoises et françaises pour l'étude des entretiens post-leçons.

5. 1. Indicateurs des pratiques d'accompagnement au Québec et en France : durée et fréquence

Pour caractériser les contextes de l'accompagnement au Québec et en France, nous avons choisi, comme discuté dans la section méthodologique de nous intéresser à des indicateurs macroscopiques tels que la fréquence des rencontres informelles et formelles. Nous présentons dans cette section ces résultats qui, selon notre approche, visent à éclairer les analyses qualitatives que nous développerons dans la section suivante. Identifier, décrire et analyser la fréquence des pratiques d'accompagnement mises en place par deux maîtres de stage tout au long du stage en responsabilité auprès de leurs stagiaires en EP au Québec et en France a été possible à partir de la synthèse des entretiens téléphoniques réalisés auprès des stagiaires tout au long du stage en responsabilité. Nous avons identifié pour chacune des semaines de stage le nombre de rencontres avec le maître de stage que le stagiaire nous rapportait ainsi que leur durée. Nous avons également inclu les entretiens post-leçons afin de situer ces derniers dans le déroulement du stage, mais aussi pour les comptabiliser dans le temps accordé aux pratiques d'accompagnement.

Le tableau III permet de situer le contexte de l'accompagnement de la stagiaire québécoise lors des sept semaines de son stage en responsabilité à partir de cinq entretiens téléphoniques.

Tableau III. Modalités temporelles des rencontres maître de stage - stagiaire pendant le stage en responsabilité au Québec 


\begin{tabular}{c|c|c|c|c|c|}
\hline \multicolumn{2}{c}{ Semaines } & $\begin{array}{c}\text { Rencontres } \\
\text { (nombre) }\end{array}$ & $\begin{array}{c}\text { Durée totale } \\
(\mathrm{min})\end{array}$ & $\begin{array}{c}\text { Entretiens post- } \\
\text { leçons (nombre) }\end{array}$ & $\begin{array}{c}\text { Durée (min) } \\
(\mathrm{min})\end{array}$ \\
\hline 1 & - & - & - & - & $\begin{array}{c}\text { Durée totale } \\
2\end{array}$ \\
3 & - & - & 1 & 23 & 23 \\
4 & - & - & 1 & 13 & 13 \\
5 & 3 & 30 & - & - & 30 \\
6 & 3 & 12 & - & - & 25 \\
7 & 3 & 45 & - & - & 45 \\
\hline Total & 12 & $2 \mathrm{~h} 44$ & 3 & 49 & 37 \\
\hline
\end{tabular}

Ces premières données nous permettent d'identifier la fréquence et la durée des rencontres maître de stage - stagiaire dans le cas québécois. Dès à présent, nous souhaitons rappeler que nous nous sommes appuyé sur des pratiques déclarées (entretiens téléphoniques dirigés avec le stagiaire) pour établir la fréquence et la durée des rencontres ce qui en constitue une limite et impose d'interpréter ces résultats avec prudence. Cependant, ces informations nous donnent de bonnes indications sur la dynamique de l'accompagnement qui a prévalu pendant ce stage de sept semaines. Au total, la stagiaire nous a dit avoir rencontré son maître de stage 12 fois et réalisé trois entretiens post-leçons. Ces derniers se sont plutôt déroulés dans la première partie du stage (semaine 2, 3 et 5) et ont eu des durées assez courtes (23 minutes pour le plus long). Nous proposerons une analyse plus approfondie de ces entretiens dans la prochaine section des résultats. Concernant les 12 rencontres rapportées par le stagiaire, elles se répartissent plutôt dans la seconde partie du stage à partir de la quatrième semaine et duraient en moyenne 14 minutes. De plus, la fréquence des rencontres a eu tendance à augmenter tout au long du stage pour passer d'aucune rencontre dans la première semaine à quatre dans la dernière. Enfin, si l'on additionne l'ensemble des rencontres rapportées par la stagiaire ( 2 h $44 \mathrm{~min}$ ) et la durée des entretiens post-leçons (49 min), le maître de stage et le stagiaire se sont rencontrés 3 h 33 min pour toute la durée du stage de sept semaines. En France, le stage en responsabilité dure neuf mois, mais pour des raisons de faisabilité, la collecte des données s'est étalée sur sept mois soit 29 semaines parce qu'elle a débuté un mois après la rentrée scolaire de septembre, pour se terminer en mai. Le tableau suivant situe le contexte de l'accompagnement du stagiaire français lors de son stage en responsabilité à partir des neuf entretiens téléphoniques.

Tableau IV. Modalités temporelles des rencontres maître de stage - stagiaire pendant le stage en responsabilité en France 


\begin{tabular}{|c|c|c|c|c|c|}
\hline Semaines & $\begin{array}{l}\text { Rencontres } \\
\text { (nombre) }\end{array}$ & $\begin{array}{l}\text { Durée totale } \\
\text { (min) }\end{array}$ & $\begin{array}{c}\text { Entretiens post-leçons } \\
\text { (nombre) }\end{array}$ & $\begin{array}{l}\text { Durée } \\
\text { (min) }\end{array}$ & Durée totale (min) \\
\hline 1 & - & - & - & - & - \\
\hline 2 & - & - & - & - & - \\
\hline 3 & 2 & 9 & 1 & 26 & 35 \\
\hline 4 & 3 & 17 & - & - & 17 \\
\hline \multicolumn{6}{|l|}{ Vacances } \\
\hline \multicolumn{6}{|l|}{ Toussaint } \\
\hline 7 & 2 & 10 & - & - & 10 \\
\hline 8 & 5 & 27 & 1 & 32 & 59 \\
\hline 9 & 2 & 8 & - & - & 8 \\
\hline 10 & 3 & 21 & - & - & 21 \\
\hline 11 & - & - & - & - & - \\
\hline 12 & 1 & 5 & - & - & 5 \\
\hline 13 & 1 & 5 & 1 & 25 & 30 \\
\hline 14 & 2 & 20 & - & - & 20 \\
\hline \multicolumn{6}{|l|}{ Vacances } \\
\hline \multicolumn{6}{|l|}{ Noël } \\
\hline 17 & 3 & 13 & - & - & 13 \\
\hline 18 & 1 & 5 & - & - & 5 \\
\hline 19 & 1 & 5 & 1 & 28 & 33 \\
\hline 20 & - & - & - & - & - \\
\hline 21 & 1 & 5 & - & - & 5 \\
\hline 22 & 1 & 5 & - & - & 5 \\
\hline \multicolumn{6}{|l|}{ Vacances } \\
\hline \multicolumn{6}{|l|}{ Hiver } \\
\hline 25 & 1 & 10 & - & - & 10 \\
\hline 26 & - & - & - & - & - \\
\hline 27 & 1 & 5 & 1 & 25 & 30 \\
\hline 28 & - & - & - & - & - \\
\hline 29 & 2 & 8 & - & - & 8 \\
\hline Total & 32 & 2 h 58 & 5 & 2 h 16 & 5 h 14 \\
\hline
\end{tabular}

Lors des 29 semaines de stage que nous avons analysées, le stagiaire nous a signalé 32 rencontres avec son maître de stage et il y a eu également cinq entretiens post-leçons qui ont duré de 25 à 32 minutes. Ces derniers sont répartis de façon équitable tout au long du stage toutes les quatre ou cinq semaines. Par contre, la fréquence des rencontres rapportées par le stagiaire est plus importante dans la première moitié du stage. Ainsi, les 
deux tiers de ces rencontres (21 sur 31 ) ont été faits dans les 14 premières semaines que nous avons observées. Dans les dix dernières semaines de stage, le maître de stage et le stagiaire se rencontraient en moyenne moins d'une fois par semaine. Enfin, si l'on additionne l'ensemble des rencontres rapportées par le stagiaire ( 2 h 58 min) et la durée des entretiens post-leçons ( 2 h 16 min), le maître de stage et le stagiaire se sont rencontré 5 h 14 min pendant les sept mois de stage en responsabilité que nous avons observé en France.

Cette première partie des résultats nous donne des indications pour comprendre la dynamique des pratiques d'accompagnement mises en place par deux maîtres de stage tout au long d'un stage en responsabilité. Une première interprétation consiste à souligner combien la dynamique qui préside aux pratiques d'accompagnement au Québec et en France est liée à la durée totale du stage, mais également à la répartition des heures dans les deux systèmes de formation ainsi qu'à la disponibilité de chacun des maîtres de stage. Au Québec, ce dernier est déchargé de cours puisque le stagiaire prend en responsabilité deux des classes. Sur une courte durée (7 semaines), les rencontres sont nombreuses, notamment dans la deuxième moitié du stage, mais ne relèvent pas toujours d'entretien post-leçon. En France, les rencontres informelles, en dehors des moments d'observation et d'entretiens post-leçons, sont plus courtes, plus éphémères et se déroulent le plus souvent dans les lieux de croisement du maître de stage et du stagiaire, puisque le premier n'est pas dégagé de ses tâches d'enseignement. La structure du stage en responsabilité, qui est spécifique à chaque système de formation, a des répercussions sur les modalités temporelles de la pratique d'accompagnement en terme de fréquence, en terme de durée et au niveau de la répartition tout au long du stage. Au Québec, les entretiens post-leçons, dans un stage en responsabilité plus intensif, ne se démarquent pas significativement en terme de durée par rapport aux rencontres informelles avec le maître de stage. De plus, à part dans la cinquième semaine de stage, il y a eu soit des entretiens post-leçons, soit des rencontres informelles, mais pas les deux en même temps. Cela laisse penser que l'accompagnement pouvait se dérouler aussi bien dans les rencontres informelles et que les entretiens post-leçons n'occupaient pas un statut particulier. En contrepartie, nous pouvons penser que les entretiens post-leçons constituent la colonne vertébrale de l'accompagnement en France, car leur fréquence rythme le stage et leur durée semble indiquer qu'ils occupent une place plus importante que les autres types de rencontres. Ce corpus de données quantifiables, relatif aux pratiques d'accompagnement, permet de contextualiser la dynamique de l'accompagnement. II ne dit cependant rien sur les contenus de cet accompagnement que 
nous allons maintenant étudier à partir de l'étude des entretiens post-leçons qui - parce qu'ils constituent les temps forts des pratiques des maîtres de stage - sont réellement le cœur de cette recherche.

5. 2. Analyse du cœur des pratiques d'accompagnement : les entretiens post-leçons Notre recherche vise à identifier, décrire et analyser le contenu des pratiques d'accompagnement mises en place par les deux maîtres de stage. Nous nous sommes centrés sur l'analyse des entretiens post-leçons qui sont une des pierres angulaires de ces pratiques. Dans un premier temps, nous avons analysé le contenu des échanges entre le maître de stage et le stagiaire. Nous avons ensuite analysé la répartition du temps de parole entre ces deux acteurs.

5. 2. 1. Analyse du contenu des entretiens post-leçons au regard de l'approche de l'écologie de la classe.

Rappelons que les contenus des entretiens post-leçons ont été mis au jour à partir des catégories relatives aux trois systèmes de tâches empruntés à l'approche de l'écologie de la classe (Siedentop, 1994) en relation avec les activités physiques enseignées par les stagiaires. Nous présentons successivement les résultats relatifs à la dyade québécoise puis française.

Tableau V Répartition du temps accordé aux systèmes de tâches dans les entretiens postleçons au Québec

\begin{tabular}{lcccc}
\hline & & $\begin{array}{c}\text { Entretien 1 } \\
\text { Handball }\end{array}$ & $\begin{array}{c}\text { Entretien 2 } \\
\text { Handball }\end{array}$ & $\begin{array}{c}\text { Entretien 3 } \\
\text { Handball }\end{array}$ \\
\cline { 3 - 5 } $\begin{array}{l}\text { Systèmes de } \\
\text { d'apprentissage (STA) }\end{array}$ & tâches & $33 \%$ & $49 \%$ & $60 \%$ \\
$\begin{array}{l}\text { Systèmes de } \\
\text { d'organisation (STO) }\end{array}$ & tâches & $15 \%$ & $37 \%$ & $15 \%$ \\
$\begin{array}{l}\text { Systèmes de } \\
\text { d'interactions sociales (STIS) }\end{array}$ & $39 \%$ & $3 \%$ & $10 \%$ \\
$\begin{array}{l}\text { Autres } \\
\text { Non lié à l'EP }\end{array}$ & $5 \%$ & $11 \%$ & $13 \%$ \\
\hline TOTAUX & $8 \%$ & $0 \%$ & $2 \%$ \\
\hline
\end{tabular}

Un premier constat, nous avons été capables de coder plus de $85 \%$ des sujets de discussion dans ces entretiens à l'aide des trois systèmes de tâches. D'autre part, le système de tâches d'apprentissage prend de plus en plus d'importance au cours des trois entretiens. En effet, il double quasiment pour passer de $33 \%$ dans le premier entretien à 
$60 \%$ dans le troisième. On peut peut-être y voir une centration progressive sur les contenus d'enseignement dans les discussions entre la stagiaire et son maître de stage au fur et à mesure que le stage avance. En contrepartie, le système de tâches d'interaction sociale occupe la place la plus importante des trois systèmes pendant le premier entretien post-leçon et diminue de façon marquée dans les deux entretiens suivants. Dans la suite de notre interprétation précédente, on peut supposer que des lacunes au sujet dusystème de tâches d'interaction sociales (STIS) ont été mises au jour, discutées et en partie comblées suite au premier entretien ce qui expliquerait une diminution du temps qui lui est accordé. Pour sa part, le système de tâches d'organisation (STO) est discuté principalement dans le second entretien, mais reste derrière le système de tâches d'apprentissage (STA). Comme les entretiens post-leçons se sont déroulés dans la deuxième semaine du stage et que l'activité est restée le handball, il se pourrait que les problèmes d'organisation aient été réglés dans la première semaine de façon autonome par la stagiaire. Enfin, sur ces trois entretiens, les discussions autour du STA représentent en moyenne $47 \%$ du temps, le STO $22 \%$ et le STIS $17 \%$.

Pour les cinq entretiens post-leçons que nous avons analysés en France, nous avons trouvé les résultats consignés au tableau VI.

Tableau VI. Répartition du temps accordé aux systèmes de tâches dans les entretiens post-leçons en France

\begin{tabular}{lccccc}
\hline & $\begin{array}{c}\text { Entretien 1 } \\
\text { Handball }\end{array}$ & $\begin{array}{c}\text { Entretien 2 } \\
\text { Badminton }\end{array}$ & $\begin{array}{c}\text { Entretien 3 } \\
\text { Badminton }\end{array}$ & $\begin{array}{c}\text { Entretien 4 } \\
\text { Volley-ball }\end{array}$ & $\begin{array}{c}\text { Entretien 5 } \\
\text { Gymnastique }\end{array}$ \\
\cline { 2 - 6 } $\begin{array}{l}\text { Systèmes de tâches } \\
\text { d'apprentissage (STA) }\end{array}$ & $52 \%$ & $48 \%$ & $32 \%$ & $48 \%$ & $60 \%$ \\
$\begin{array}{l}\text { Systèmes de tâches } \\
\text { d'organisation (STO) }\end{array}$ & $27 \%$ & $27 \%$ & $47 \%$ & $31 \%$ & $15 \%$ \\
$\begin{array}{l}\text { Systèmes de tâches } \\
\text { d'interactions sociales }\end{array}$ & $15 \%$ & $14 \%$ & $13 \%$ & $15 \%$ & $19 \%$ \\
$\begin{array}{l}\text { (STIS) } \\
\text { Autres }\end{array}$ & $4 \%$ & $10 \%$ & $4 \%$ & $6 \%$ & $4 \%$ \\
Non lié à l'EP & $2 \%$ & $1 \%$ & $4 \%$ & $0 \%$ & $2 \%$ \\
\hline TOTAUX & $100 \%$ & $100 \%$ & $100 \%$ & $100 \%$ & $100 \%$
\end{tabular}

Dans les cinq entretiens, nous observons une certaine constance dans la proportion de temps accordée au STIS (autour de $15 \%$ ). Pour sa part, le STO a subit de gros écarts passant de $47 \%$ (entretien 3) à $15 \%$ (entretien 5). Le STA a, lui aussi, grandement varié 
en passant de $32 \%$ (entretien 3) à $60 \%$ (entretien 5). Cependant, ce STA est resté relativement proche de $50 \%$ du temps pour les trois autres entretiens. Sur les cinq entretiens, le STA représente en moyenne $48 \%$ du temps, le STO $29 \%$ et le STIS $15 \%$. Les résultats du cas québécois montrent que le STIS tombe de $39 \%$ dans l'entretien 1 à 3 $\%$ dans l'entretien 2. Dès à présent, certaines questions se posent : la stagiaire a-t-elle réglé entre l'entretien 1 et 2 des problèmes majeurs de gestion du comportement des élèves en classe ? D'autre part, le STA prend de plus en plus de place dans les entretiens post-leçons entre le maître de stage et le stagiaire au fur et à mesure que le stage avance. Peut-on y voir le signe que la stagiaire et le maître de stage sont progressivement en mesure de s'intéresser davantage aux réels apprentissages des élèves ? En France, le STO passe de $47 \%$ de l'activité badminton (entretien 3) à $15 \%$ pour la gymnastique (entretien 5). Quel est l'impact de l'activité physique enseignée sur les thématiques traitées dans les entretiens post-leçons ? Les spécialités sportives déclarées du stagiaire et du maître de stage ont-elles une influence sur cette répartition ? Qui du maître de stage ou du stagiaire s'exprime le plus au sujet de chacun de ces systèmes de tâches?

Ces premiers résultats nous permettent d'ores et déjà d'avancer certaines pistes de comparaison entre les deux cas. En effet, il apparaît qu'autant chez la dyade du Québec que chez la dyade de la France, le STA est la thématique la plus traitée dans l'ensemble des entretiens (excepté le premier entretien post-leçon québécois). De plus, STO arrive au second plan suivi du STIS. Cette tendance générale s'observe indépendamment de l'activité enseignée et de la durée des entretiens post-leçons. Toutefois, même si cet ordre général d'apparition est observable, des variations importantes apparaissent entre les deux cas, mais également au sein d'un même cas. Par exemple, au Québec, le STA augmente progressivement au cours des trois entretiens. En contrepartie, le STA varie au cours des cinq entretiens en France même si une augmentation est observée dans les trois derniers.

Dans le but d'éclairer nos analyses, nous avons mesuré le temps de parole respectif du maître de stage et du stagiaire dans les entretiens post-leçons avant de le mesurer pour chacun des systèmes de tâches.

5. 2. 2. Un indicateur pour saisir la dynamique des entretiens post-leçons : la répartition du temps de parole maitre de stage - stagiaire Nous avons mesuré pour chacun des entretiens post-leçons la répartition du temps de parole entre le maître de stage et le stagiaire dans les trois entretiens du cas québécois et les cinq du cas français. Ces résultats sont présentés dans la figure 1. 


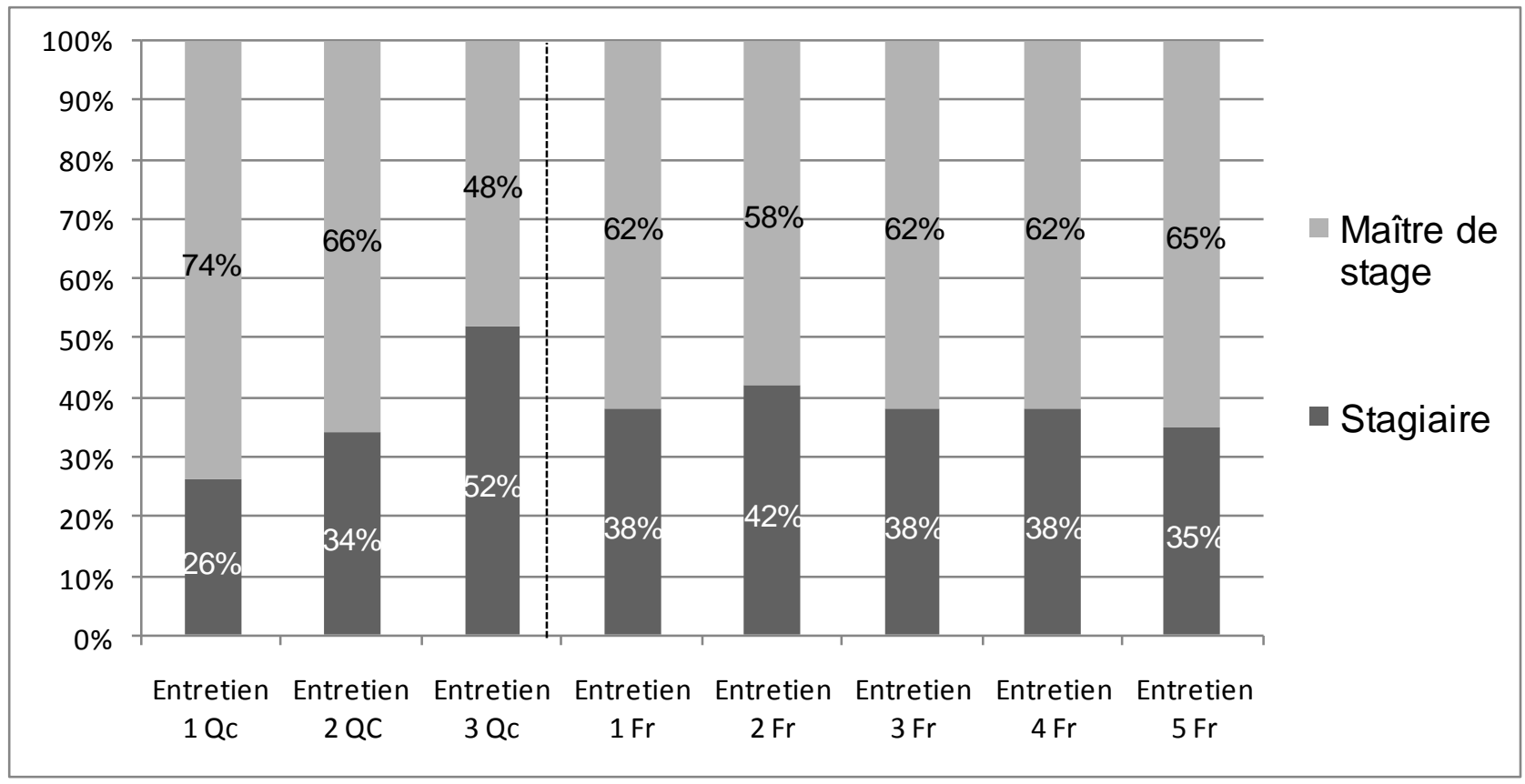

Figure 1 : Répartition du temps de parole dans les entretiens post-leçons au Québec (Qc) et en France (Fr)

La figure précédente fait apparaître que le stagiaire québécois prend de plus en plus la parole dans les entretiens post-leçons au fur et à mesure que le stage en responsabilité avance. Dans le dernier entretien, le stagiaire parle plus que son maître de stage.Du côté de la dyade française, la répartition du temps de parole entre le maître de stage et son stagiaire est relativement stable au cours des cinq entretiens que nous avons analysés et se situe en moyenne autour de 62 \% pour le maître de stage et de 38 \% pour le stagiaire. La spécificité des contextes peut-elle expliquer les différences de dynamique observées entre les cas québécois et français ? L'enseignement des contenus en lien avec le handball au Québec par rapport à des contenus relatifs à quatre activités physiques différentes en France a peut-être eu une influence sur la dynamique des entretiens postleçons. Au Québec la stagiaire prend de plus en plus la parole au cours des trois entretiens alors que la répartition reste constante en France. Ce type de répartition de la parole est-il identique pour chacun des systèmes de tâches traitées dans les entretiens post-leçons ? Dans le but de mettre en tension les résultats des deux figures précédentes et ceux issus des analyses du contenu des entretiens, nous avons calculé la répartition du temps de parole entre le maître de stage et le stagiaire pour chacun des trois systèmes de tâches.

Nous nous sommes rendu compte qu'autant au Québec qu'en France, les stagiaires prennent plus la parole (par rapport aux résultats présentés dans la figure 1) lorsqu'il est question du STA (3\% de plus au Québec et $9 \%$ en France). Par contre, les maîtres de stage parlent plus lorsqu'il est question du STO (2\% de plus au Québec et $12 \%$ en 
France). Enfin, concernant le STIS, le temps de parole des stagiaires est relativement similaire par rapport aux moyennes présentées dans la figures 1 (2\% de plus au Québec et $1 \%$ de moins au Québec). Ces résultats montrent que les stagiaires ont tendance à prendre plus de leadership dans les entretiens post-leçons lorsque les contenus d'enseignement et les apprentissages des élèves sont en jeu (STA). De leur côté, cette dynamique s'observe chez les maîtres de stage au sujet de l'organisation de la séance d'EP. Peut-on en déduire que les maîtres de stage se tiennent responsables de l'organisation de la classe tout en laissant aux stagiaires la responsabilité des contenus à enseigner en EP dans le stage en responsabilité ? Un autre volet visant une analyse plus fine (d'ordre didactique) de ces échanges entre les maîtres de stage et les stagiaires est actuellement en cours et viendra compléter les résultats que nous présentons dans cet article.

Ces différents résultats répondent au premier objectif qui était d'identifier, de décrire et d'analyser la fréquence et le contenu des pratiques d'accompagnement mises en place par deux maîtres de stage tout au long du stage en responsabilité auprès de leurs stagiaires en éducation physique au Québec et en France. Nous avons en effet montré que la fréquence des rencontres augmentait tout au long du stage chez la dyade québécoise alors que la tendance semblait contraire chez la dyade française. Cependant, il n'y a pas eu d'entretiens post-leçons dans les deux dernières semaines de stage dans le cas québécois. D'autre part, nous avons été en mesure d'identifier la durée totale des rencontres entre un maître de stage et son stagiaire tout au long de leurs stages. Enfin, nous avons identifié, décrit et analysé le contenu des pratiques d'accompagnement en nous centrant sur l'étude des entretiens post-leçons à l'aide des trois systèmes de tâches (STA, STO, STIS).

\section{Discussion sur l'empreinte des contextes de stage sur les pratiques d'accompagnement}

Nous cherchions à travers l'analyse comparative des pratiques d'accompagnement des stagiaires au Québec et en France à identifier, décrire et analyser les points communs et les différences entre les pratiques mises en place par les maîtres de stage en considérant la spécificité des contextes de formation dans lesquelles elles étaient mises en jeu. Nous allons dans un premier temps nous centrer sur les points communs à ces pratiques avant de discuter leur particularité. Pour mener cette discussion, nous mettrons nos résultats en tension avec ceux d'études précédentes.

6. 1. Au-delà des contextes, des modalités d'accompagnement fort semblables 
L'étude de la fréquence des pratiques d'accompagnement tout au long du stage nous a permis de faire ressortir qu'autant chez la dyade française que québécoise, les entretiens post-leçons n'ont pas lieu chaque semaine, mais qu'ils sont dispersés tout au long du stage. En effet, ces rencontres ont eu lieu dans les semaines 2, 3 et 5 au Québec et dans les semaines 3, 8, 13, 19 et 27 en France. II apparaît également que ces rencontres ont une durée relativement courte (presque toutes en dessous de 30 minutes et certaines ne durent même moins de 15 minutes au Québec). Rappelons que nous avons volontairement laissé les maîtres de stage et les stagiaires libres dans l'organisation de ces rencontres. Les entretiens post-leçons qui sont considérés comme des périodes d'échange et d'accompagnement essentielles dans la dynamique d'un stage dans la littérature ne semblent donc pas recevoir la même considération de la part des acteurs de ces stages. Dans ce cas-ci, le manque de temps ne semble pas être un facteur déterminant, car le maître de stage québécois était totalement déchargé de son enseignement pendant le stage en responsabilité. $Y$ aurait-il lieu de creuser du côté de la sélection et de la formation des maîtres de stage ? Quel sens les maîtres de stage et les stagiaires donnent-t-ils à ces pratiques d'accompagnement?

D'autre part, l'analyse des entretiens post-leçons fait ressortir que les thématiques qui reviennent en premier sont reliées directement à la matière enseignée en EP et les apprentissages que le stagiaire a l'intention de faire réaliser aux élèves pendant leur participation aux activités proposées (STA). Cette dynamique est observée autant au Québec qu'en France avec une répartition moyenne entre les trois systèmes de tâches relativement proche, et ce, indifféremment de l'activité enseignée. En effet, en moyenne les entretiens post-leçons québécois se répartissaient à $47 \%$ dans le STA, $22 \%$ pour le STO et $17 \%$ pour le STIS et pour les français, $48 \%$ pour le STA, $29 \%$ pour le STO et 15 $\%$ pour le STIS. Ces résultats viennent nuancer ceux d'études précédentes qui soulignaient le fait que les discussions au sujet des contenus d'enseignement en EP étaient reléguées au second plan derrière l'organisation et la gestion de classe dans les entretiens post-leçons (Dugal \& Amade-Escot, 2004). Doit-on en déduire que les thématiques en lien avec la matière enseignée en EP et les apprentissages des élèves sont au cœur des préoccupations dans les échanges entre les maîtres de stage et les stagiaires dans les stages en responsabilité ? Ce stage est le dernier avant une autonomie complète du futur enseignant, y-aurait-il également une centration sur le STA si l'on analysait les pratiques d'accompagnement mises en place dans des stages plus tôt dans la formation? 
Enfin, une analyse plus approfondie de la répartition moyenne du temps de parole entre stagiaires et maître de stage par rapport au système de tâches nous a permis de faire apparaître que les stagiaires prenaient davantage la parole lorsque la discussion portait sur le STA alors que les maîtres de stage prenaient plus la parole lorsqu'il était question du STO. Peut-on voir des effets de la formation initiale des stagiaires dans ces résultats et une centration sur les aspects organisationnels de la part des acteurs du terrain ? Ces derniers considèrent peut-être qu'il s'agit là de leur domaine d'expertise de par leur expérience que ne possède pas le stagiaire. Est-ce que sur le plan du STA, les maîtres de stage se sentent moins au fait des dernières évolutions sur les contenus, au contraire du stagiaire, « frais émoulu » de la formation (Dugal \& Amade-Escot, 2004) ?

6. 2. Des spécificités marquées par les systèmes de formation nationaux

Le premier aspect que nous avons associé à des pratiques spécifiques concerne la répartition de la fréquence des rencontres hebdomadaires qui augmente tout au long du stage pour atteindre quatre rencontres dans la septième semaine au Québec alors que 21 des 31 rencontres se situent dans la première partie du stage en France. D'autre part, la durée des rencontres rapportées sur une semaine est trois fois plus importante au Québec (18 min par semaine) qu'en France (6 min par semaine). II faut toutefois se souvenir que les stages ont eu une durée différente entre le Québec (7 semaines) et la France (9 mois). Enfin, le nombre d'entretiens post-leçons pour un stage en responsabilité diffère avec un total de trois entretiens pour le Québec et cinq pour la France. Une interprétation possible de ces résultats peut être faite à partir de la durée des deux stages, de la répartition des journées de stage dans la semaine et du statut du maître de stage. En effet, le stagiaire québécois est présent pendant sept semaines, tous les jours et son maître de stage est totalement déchargé de son enseignement. Pour sa part, le stagiaire français est présent deux jours par semaine sur son lieu de stage ( 8 heures au total) pendant 9 mois et son maître de stage continue d'enseigner pendant 21 heures par semaine. Ces éléments de contextes peuvent expliquer le fait que la durée des rencontres par semaine soit plus grande au Québec qu'en France.

Cependant, les institutions québécoises, en libérant totalement le maître de stage de son enseignement, semblent clairement donner des occasions d'accompagnement du stagiaire plus importantes. Pourtant, les entretiens post-leçons au Québec sont moins importants en nombre et en durée qu'en France. D'autre part, l'analyse du temps de parole respectif entre le maître de stage et le stagiaire dans les entretiens post-leçons a montré une nette augmentation de la prise de parole de la stagiaire au Québec alors que la répartition est restée relativement stable dans le cas français. Ces différences peuvent 
provenir des différences de style d'accompagnement et de leur orientation vers une posture directive du maître de stage ou plutôt orientée vers les stagiaires (Bertone et al., 2006). Cependant, nous n'avons pas encore de données assez solides sur ce sujet pour proposer une interprétation plus incisive.

Le dernier élément spécifique concerne la répartition entre les trois systèmes de tâches pour chacun des entretiens post-leçons. En effet, nous avons observé une variation entre ces répartitions, une piste d'interprétation que nous pouvons avancer concerne le lien entre l'activité physique enseignée et les spécialités sportives du maître de stage et du stagiaire. D'autre part, le visionnement des séquences vidéo nous donne d'autres pistes d'interprétation à explorer. En effet, lors de la séance de gymnastique (entretien 5 français), le matériel était déjà présent et installé dans le gymnase ce qui peut expliquer le peu de discussions au sujet du STO pour cette séance.

Notre cadre théorique actuel ne nous permet pas d'aller plus loin dans l'analyse de ces échanges. L'avantage de l'approche de l'écologie de la classe à l'aide des systèmes de tâche est qu'elle prend en compte l'ensemble des évènements qui ont lieu dans la classe. Ces catégories se sont avérées très utiles pour rendre compte des thématiques traitées dans les entretiens post-leçons entre le maître de stage et le stagiaire. Cependant, ce cadre théorique ne nous permet pas d'analyser plus en profondeur les échanges et les sujets réellement en jeu notamment au sein du système de tâches d'apprentissage.

6. 3. L'intérêt de ces résultats par rapport à la littérature existante

Notre recherche portait sur un stage de sept semaines au Québec et de neuf mois en France. Tannehill et Zakrajsek (1988) ont réalisé une étude portant sur huit semaines auprès de 18 maîtres de stage et de leurs stagiaires. Ces auteurs ont observé que la fréquence des rencontres maître de stage - stagiaire diminuait significativement au cours des huit semaines. De notre côté, nous avons pu observer que la répartition de la fréquence des rencontres variait entre le Québec (où elle augmente) et la France (où elle diminue) tout au long du stage. D'autre part, Tannehill et Zakrajsek (1988) ont également observé que les maîtres de stage ne rencontraient plus leur stagiaire dans les dernières semaines en fin de stage.

Nous avons également observé ce phénomène en France. Ces résultats suggèrent que la durée du stage n'est pas un facteur qui détermine la fréquence des rencontres maître de stage - stagiaire au cours d'un stage en responsabilité. D'autre part, plusieurs recherches portant sur les pratiques d'accompagnement ont montré que les maîtres de stage accompagnaient prioritairement leurs stagiaires sur la gestion de classe, l'organisation et la gestion de l'indiscipline (Banville, 2006; Dugal et Amade-Escot, 2004). Nous avons pu 
identifier ces catégories que nous avons associées au STIS dans les entretiens postleçons. Cependant, contrairement à ces recherches, nous avons trouvé que le sujet qui était le plus discuté était en lien avec les activités reliées directement à la matière enseignée en EP et les apprentissages que le stagiaire a l'intention de faire réaliser aux élèves pendant leur participation aux activités proposées (STA). Cette tendance a été observée à partir de pratiques effectives de conseil pédagogique autant au Québec qu'en France.

\section{Conclusion}

L'étude des pratiques d'accompagnement mises en place par deux maîtres de stage, l'un au Québec et l'autre en France, répond à certaines interrogations, mais pose aussi une série de questions. Tout d'abord, nous avons montré que la fréquence et la durée des rencontres entre un maître de stage et son stagiaire variaient d'un contexte à un autre. Cependant, il nous semble que la durée est relativement faible pour que des pratiques d'accompagnement de stagiaires soient porteuses d'un réel développement des compétences professionnelles. II est important de rappeler que notre recherche n'a porté que sur un maître de stage et son stagiaire au Québec et un maître de stage et son stagiaire en France ce qui impose d'interpréter ces résultats en considérant ces limites. D'autre part, notre cadre théorique d'analyse des entretiens post-leçons nous a donné de bonnes indications sur la dynamique générale de ces entretiens. Malheureusement, même si les trois systèmes de tâches empruntés à l'approche de l'écologie de la classe nous permettent de bien rendre compte des sujets traités dans les entretiens post-leçons, ils ne sont pas assez fins aux plans théorique et conceptuel pour porter un éclairage sur les pratiques d'accompagnement ayant lieu dans chacun des trois systèmes de tâche. Nous travaillons actuellement sur l'articulation d'un second cadre théorique plus spécifique d'ordre didactique nous permettant d'analyser les échanges entre le maître de stage et le stagiaire spécifiquement dans le STA. Nos recherches nous ont conduits aux travaux en didactique sur l'action conjointe du professeur et des élèves développées par Sensevy et Mercier (2007) qui pourraient s'articuler à l'approche de l'écologie de la classe et aller plus loin dans l'analyse des échanges maître de stage - stagiaire. II nous semble que l'étude des pratiques d'accompagnement des stagiaires représente un véritable enjeu pour faire avancer la formation pratique des enseignants d'EP, mais aussi la formation des maîtres de stage. 


\section{Bibliographie}

Altet, M. (1994). La formation professionnelle des enseignants : Analyse des pratiques et situations pédagogiques (1e éd ed.). Paris: Presses universitaires de France.

Banville, D. (2006). Analysis of exchanges between novice and cooperating teachers during internships using the NCATE/NASPE standards for teacher preparation in physical education as guidelines. Research Quarterly for Exercise and Sport, 77(2), 208.

Barbier, J. (2006). Les voies nouvelles de la professionnalisation. In Y. Lenoir, \& M. Bouillier-Oudot (Eds.), Savoirs professionnels et curriculum de formation (pp. 6781). Québec: Les presses de l'Université Laval.

Bertone, S., Chalies, S., Clarke, A., \& Méard, J. (2006). The dynamics of interaction during post-lesson conferences and the development of professional activity : Study of a pre-service physical education teacher and her co-operating teacher. Asia-Pacific Journal of Teacher Education, 34(2), 245-264.

Brien, R. (1994). Science cognitive et formation. Sainte-Foy : Presses de l'Université du Québec.

Bronfenbrenner, U. (1980). The ecology of human development: Experiments by nature and design. United States; Etats-unis.

Bronfenbrenner, U. (1981). L'écologie expérimentale de l'éducation. In A. Beaudot, A. Gonnin-Lafond \& D. Zay (Eds.), Sociologie de l'école. Pour une analyse de l'établissement scolaire (pp. 21-50). Paris: Bordas.

Bru, M. (1993). L'enseignant organisateur des conditions d'apprentissage. In J. Houssaye (Ed.), La pédagogie, une encyclopédie pour aujourd'hui (pp. 103-117). Paris: ESF.

Bulletin officiel du Ministère de l'Éducation nationale. (2007). Cahier des charges de la formation des maîtres en IUFM (No. 1 du 4 janvier 2007). Paris: Ministère de l'Éducation nationale.

Bulletin officiel du Ministère de l'Éducation nationale (2007). Mise en oeuvre du cahier des charges de la formation des maîtres ( ${ }^{\circ} 9$ du 1er mars 2007). Paris: Ministère de l'Éducation.

Chalies, S. \& Durand, M. (. (2000). L'utilité discutée du tutorat en formation initiale des enseignants : Formes et dispositifs de la professionnalisation. Recherche et Formation, 35, , 145-180.

Clarke, A. (2006). The nature and substance of cooperating teacher reflection. Teaching \& Teacher Education: An International Journal of Research and Studies, 22(7), 910. 
COFPE. (2005). La formation en milieu pratique : De nouveaux horizons à explorer.

Québec: gouvernement du Québec.

COFPE. (2006). La formation en milieu de pratique : De nouveaux horizons à explorer. Québec: gouvernement du Québec.

Coleman, M. M., \& Mitchell, M. (2000). Assessing observation focus and conference targets of cooperating teachers. Journal of Teaching in Physical Education, 20(1), 40.

Doyle, W. (1979). Classroom effects. Theory into Practice, 18(3), 138.

Doyle, W. (1981). Research on classroom contexts. Journal of Teacher Education, 32(6), 3-6.

Doyle, W. (1986). Classroom organization and management. In M. Wittrock (Ed.), Handbook of research on teaching (Macmillan ed., pp. 392-431). New-York: MacMillan.

Dugal, J.P., \& Amade-Escot, C. (2004). Formation au conseil et développement professionnel des conseillers pédagogiques. Recherche coopérative et savoirs didactiques. Recherche et Formation, 46, 97-116.

Hastie, P. A., \& Siedentop, D. (2006). The classroom ecology paradigm. In D. Kirk, D. Mac Donald \& M. O'Sullivan (Eds.), The handbook of physical education (pp. 214-225). Londre: Sage.

Hastie, P. A., Sinelnikov, O. A., Brock, S. J., Sharpe, T. L., Eiler, K., \& Mowling, C. (2007). Kounin revisited: Tentative postulates for an expanded examination of classroom ecologies. Journal of Teaching in Physical Education, 26(3), 298-309.

Hastie, P., \& Siedentop, D. (1999). An ecological perspective on physical education. European Physical Education Review, 5(1), 9.

Kounin, J. S. (1970). Discipline and group management in classrooms. New York: Holt, Rinehart and Winston.

Lacoste, J., Loarer, C., \& Monnanteuil, F. (2007). Le stage en responsabilité dans la formation initiale des professeurs. No. 2007-027. Paris: IGEN, IGAENR.

Leclerc, C. (2004). Le formateur d'enseignants, entre directivité et réflexivité. In J. Astolfi (Ed.), Savoirs en action et acteurs de la formation (pp. 111-128). Rouen: Publications de l'université de Rouen.

Maxwell, J. A. (2005). Qualitative research design: An interactive approach. (2nd ed.). Thousand Oaks: Sage Publications.

McCaughtry, N., Tischler, A., \& Flory, S. B. (2008). The ecology of the gym: Reconceptualized and extended. Quest, 60(2), 268-289. 
Ministère de l'Éducation des loisirs et du sport. (2001). La formation à l'enseignement.

Les orientations, les compétences professionnelles. Québec: gouvernement du Québec.

Ministère de l'Éducation des loisirs et du sport. (2002). Encadrement des stagiaires de la formation à l'enseignement : Rapport d'évaluation de programme. Québec: gouvernement du Québec.

Ministère de l'Éducation nationale. (2002). Enseigner, un métier pour demain. Paris: Ministère de l'Éducation nationale.

Ministère de l'Éducation nationale. (2008). Livre vert sur l'évolution du métier d'enseignant. Paris: Ministère de l'Éducation nationale.

Ria, L., Sève, C., Durand, M., \& Bertone, S. (2004). Indétermination, contradiction et exploration: Trois expériences typiques des enseignants débutants en éducation physique. Revue des Sciences de l'Éducation, 30(3), 535-554.

Roux-Pérez, T. (2006). Représentations du métier d'enseignant et rapport à la formation chez les étudiants en STAPS: Une identité professionnelle en construction. Revue STAPS, 73, 57-68.

Sensevy, G., \& Mercier, A. (2007). Agir ensemble : L'action didactique conjointe du professeur et des élèves. Rennes: Presses universitaires de Rennes.

Serres, G., Ria, L., Adé, D., \& Sève, C. (2006). Apprend-on vraiment à intervenir en formation initiale ? Prémisses du développement de l'activité professionnelle dans les dispositifs de formation en alternance. Revue STAPS, 72, 9-20.

Siedentop, D. (1994). Apprendre à enseigner l'éducation physique. Montréal: G. Morin.

Siedentop, D. (2002). Ecological perspectives in teaching research. Journal of Teaching in Physical Education, 21(4), 427-440.

Stake, R. E. (2000). Case studies. In N. K. Denzin, \& Y. S. Lincoln (Eds.), Handbook of qualitative research (pp. 435-454). London: Sage publication.

Tannehill, D., \& Zakrajsek, D. (1988). What's happening in supervision of student teachers in secondary physical education. Journal of Teaching in Physical Education, 8(1), 1.

Tinning, R. (2002). Engaging siedentopian perspectives on content knowledge for physical education. Journal of Teaching in Physical Education, 21(4), 378-391.

Tinning, R., \& Siedentop, D. (1985). The characteristics of tasks and accountability in student teaching. Journal of Teaching in Physical Education, 4(4), 286-299.

Tousignant, M. (1982). Analysis of the task structures in secondary physical education classes. Unpublished Thèse de doctorat, The Ohio State University, Columbus. 
Tousignant, M., \& Siedentop, D. (1983). A qualitative analysis of task structures in required secondary physical education classes. Journal of Teaching in Physical Education, 3(1), 47-57.

Van der Maren, J. (1996). Méthodes de recherche pour l'éducation (2e éd.). Montréal : Presse de l'Université de Montréal.

Wittorski, R. (2004). Savoir d'action, compétences et professionnalisation. In J. Astolfi (Ed.), Savoir en action et acteurs de la formation (pp. 51-76). Rouen : Publications de l'université de Rouen.

\section{Liste des acronymes}

BOEN : Bulletin officiel de l'éducation nationale (France)

CAPEPS : Certificat d'aptitude au professorat d'éducation physique et sportive (France)

COFPE : Conseil d'orientation de la formation du personnel enseignant (Québec)

EP : Éducation physique

IUFM : Institut universitaire de formation des maîtres (France)

MELS : Ministère de l'Éducation des loisirs et du sport (Québec)

MEN : Ministère de l'Éducation nationale (France)

STA : Système de tâches d'apprentissage

STIS : Système de tâches d'interactions sociales

STO : Système de tâches d'organisation 